\title{
Global Longitudinal Strain Monitoring to Guide Cardioprotective Medications During Anthracycline Treatment
}

\author{
Thomas H. Marwick ${ }^{1,2}$ (D)
}

Accepted: 6 January 2022 / Published online: 3 March 2022

(c) The Author(s) 2022

\begin{abstract}
Purpose of the Review Anthracycline chemotherapy carries a risk of myocardial dysfunction and heart failure even at relatively low doses, and the clinical prediction of cancer treatment-related cardiac dysfunction (CTRCD) is inexact. Careful imaging or biomarker surveillance during chemotherapy can identify CTRCD before the development of heart failure. Currently, this surveillance is performed using ejection fraction (EF). While this is a reliable and reproducible test with three-dimensional techniques, the most widely used imaging technique is two-dimensional echocardiography, for which EF measurements have broad confidence intervals.

Recent Findings The use of global myocardial strain (GLS) provides a more reliable and reproducible means of assessing global cardiac function and shows meaningful changes before a significant change of EF. Observational studies have shown that although absolute measurements of GLS, both at baseline and during therapy, are predictive of CTRCD risk, the most reliable approach is to assess the change of GLS with therapy - a meaningful relative change of $10-15 \%$ being significant. A clinical trial comparing GLS to EF surveillance did not show a significant change of EF in the overall study group, but did show that patients managed with a the GLS-guided approach were less likely to develop a meaningful change of cardiac function to an abnormal level. In at-risk patients, there is good evidence for the protective value of neurohormonal antagonists and statins: the use of GLS enables these benefits to be directed to those most likely to benefit, while minimizing their use in the majority of people, who do not need them. Summary Although GLS requires an element of training and efforts to ensure uniformity, it has proven to be a feasible, robust, and reproducible technique, ready for wide adoption.
\end{abstract}

Keywords Global longitudinal strain · Cardioprotective medications · Anthracycline treatment

\section{Introduction}

The increasing survival from cancer is leading to a huge cohort of cancer survivors [1]. Among these individuals, the development of heart failure (HF) during follow-up is a significant source of morbidity. The prognostic implications of HF due to chemotherapy seem to be worse than most other types of HF [2].

This article is part of the Topical collection on Cardio-oncology

Thomas H. Marwick

tom.marwick@baker.edu.au

1 Baker Heart and Diabetes Institute, PO Box 6492, Melbourne, Victoria 3004, Australia

2 Baker Department of Cardiometabolic Health, University of Melbourne, Melbourne, Victoria, Australia
Prevention and early recognition may avoid the scenario of the successfully treated cancer patient succumbing from HF.

\section{Defining Cancer Treatment-Related Cardiac Dysfunction (CTRCD)}

The condition that we are seeking to avoid is the development of HF in cancer survivors. While this may occur acutely, it is more commonly detected years after chemotherapy. Of course, awaiting the clinical presentation of HF is not a prudent approach to this diagnosis, because it takes so long to develop and because the patients at that stage have disease that is too far advanced make a therapeutic impact. Consequently, the definition of CTRCD is based upon a meaningful drop of ejection fraction $(10 \%$ 
if asymptomatic, $5 \%$ if symptomatic) to below the normal range, albeit with some differences in exact definitions [3].

The cause of LV dysfunction in cancer patients is inherently multifactorial, with significant contributions from not only chemotherapy, but also risk factors that are shared between cancer and cardiac disease [4]. Nonetheless, patients treated with potentially cardiotoxic chemotherapy represent an important group for surveillance both acutely at the time of chemotherapy, as well as during long-term follow-up.

CTRCD may arise from exposure to cytotoxic agents or biological agents. While the classification into types I and II cardiotoxicity, respectively, has been criticized as being too simplistic, it has some value. CTRCD from biological agents is usually reversible, and uncommon - most studies suggest frequencies of $2 \%$ with lapatinib, imatinib, trametinib, and bevacizumab, although up to $11 \%$ is reported with sunitinib, and up to $27 \%$ with trastuzumab [5], possibly reflecting other influences. In contrast, myocardial injury due to cytotoxic agents, related to oxidative stress, free radical formation, and cell death, is associated with ultrastructural changes at biopsy, and is usually irreversible. CTRCD may occur at three stages in relation to chemotherapy - (i) acutely (a dose-dependent and usually reversible decline in LV function during/ after infusion), (ii) subacutely (a rare, myocarditislike presentation with edema, wall thickening, and diastolic dysfunction within a few weeks), and (iii) late (usually in 1st year but occurring in up to 10-20 years, with the dose-response influenced by age, sex, underlying CVD, hypertension, and smoking) [5]. As the development of HF or LV dysfunction is not purely a reflection of chemotherapy, but is also influenced by the background risk factor milieu, possible direct effects of cancer on the heart, and the impact of other treatments including radiotherapy, this needs to be approached on a probabilistic rather than a deterministic basis.

\section{Clinical Prediction}

A Bayesian approach is necessary whenever imaging is used in risk assessment, and patients over the age of 65 , those with previous anthracycline use and radiotherapy, those with coronary or other heart disease, and hypertension are particularly at risk of developing anthracycline-related cardiotoxicity [6]. The development of LV dysfunction and cardiotoxicity is dose-dependent - the frequency of HF attributable to anthracyclines, at least acutely, has decreased from the original report by Von Hoff [7]. Paradoxically, patients administered low doses of anthracycline account for the majority of cases, as although the rate of occurrence is low, these account for a very large number of patients, who are at some risk, even at doses $<250 \mathrm{mg} / \mathrm{m}^{2}$ [8]. The use and dose of anthracycline has been incorporated into various risk prediction tools, but although these do identify the spectrum of risk, they have been unable to reliably identify a very low risk group, who could forego imaging $[9,10]$.

The fundamental need for HF surveillance in this group is a solution that will work in large numbers of people (which has implications for cost and availability), at a high level of accuracy (taking note of the risks of misdiagnosis), at a high level of test-retest consistency, and irrespective of differing baseline risk. Three successive stages precede the development of HF - an initial phase where the causative pathway is identified, abnormal myocardial deformation, and asymptomatic LV dysfunction (Table 1). The use of more specific and less sensitive tests may detect disease at a late stage, whereas more sensitive and less specific tests have the potential of detecting early disease, but at the risk of mislabeling patients as having disease.

\section{Use of Ejection Fraction to Guide Therapy}

Ejection fraction (EF) is part of the current definition of CTRCD, and is uniformly referenced in the guidelines [3, 11]. However, EF is not without its problems. EF is a useful marker of prognosis in patients with HF, particularly with an
Table 1 Identification of CTRCD at various clinical stages before the development of $\mathrm{HF}$

\begin{tabular}{llll}
\hline Clinical phase & & Imaging & Laboratory tests \\
\hline Asymptomatic LVD & & EF <53\% & BNP \\
Abnormal deformation & & Abnormal LV strain & BNP \\
Causative factors & Myocyte injury & CMR & HsTn \\
& Oxidative stress & & MPO \\
& Fibrosis & & ST-2, galectin \\
& Inflammation & & CRP, IL-6 \\
& MicroRNA & & miR-1, miR-29b, miR499 \\
\hline
\end{tabular}

Legend: $B N P$, brain natriuretic peptide; $I L$, interleukin; $M P O$, myeloperoxidase 
$\mathrm{EF}<40 \%$, but its association with outcome in individuals with preserved EF is rather poor. The diagnosis of CTRCD is based upon a meaningful reduction of $\mathrm{EF}$ to below the normal range, and there are challenges to both defining normal and defining reduction. The ability to interpret change can be confounded by alterations of loading conditions, implying that it may change despite the presence of stable myocardial function if BP changes in the course of serial follow-up. High and low heart rate, mitral regurgitation, and left bundle branch block all provide technical challenges.

Two-dimensional echocardiography is the most widely used technique for surveillance, and calculations of LV volumes using this technique are dependent on geometry, with changes from one visit to the next being influenced by different imaging planes. These relatively common differences in imaging planes lead to wide confidence intervals for the detection of sequential changes - absolute EF differences of up to $10 \%$ cannot be attributed to a change of myocardial status and may simply reflect measurement variation [12]. The definition of normal with different imaging modalities is different [13], and both sex and ethnic background influence findings [14].

EF may be calculated without geometric assumptions when a full 3-D data set is obtained, most commonly using 3D echocardiography or cardiac magnetic resonance. In contrast to the $10 \%$ confidence intervals of $2 \mathrm{D}$ echo, a meaningful change of 3D-EF is 5-6\% [15]. However, although $3 \mathrm{D}-\mathrm{EF}$ is recommended in guidelines, it is often not performed because of inadequate imaging windows and suboptimal image quality.

EF is widely assessed at baseline in patients undergoing anthracycline chemotherapy, with a recent data-linkage of chemotherapy for breast cancer with administrative data showing its use in $>85 \%$ at baseline. In contrast, only about $50 \%$ of patients have an echocardiogram performed during follow-up, and fewer than $50 \%$ have monitoring is recommended in guidelines $[16,17]$.

\section{Use of Strain to Guide Therapy}

Technical Considerations Myocardial strain measures the magnitude of deformation of a defined length of myocardium during each cardiac cycle, referenced to the original length. Because the myocardium shortens, this is classically described as a negative number, but when strain is averaged in all myocardial segments (global longitudinal strain, GLS), it is always negative, and therefore can reasonably be expressed without a negative sign (recently coined "global longitudinal shortening") [18]. This certainly facilitates communication with oncologists and other medical specialists, who may be unfamiliar with the derivation of this parameter.
The initial calculation of strain was performed using color tissue Doppler, which provided high temporal resolution, but had the disadvantage of being susceptible to noise, dependent on alignment with the Doppler beam, and being quite challenging to measure. However, the development of speckle strain, now two decades ago, has made the assessment of strain more feasible, reliable, and reproducible. Initial problems relating to inconsistencies of measured GLS by different machines, attributable to different methods of post-processing between manufacturers, have largely been addressed following a concordance process. The variability of strain between systems is now analogous to the variability of other common measurements such as LV dimensions [19•]. A number of studies have defined the normal range of strain in volunteers - a recent meta-analysis showed that a normal GLS is $>18 \%$ [20], but the interval between 16 and $18 \%$ should be considered borderline, in part because of the load-dependence of strain.

GLS has a well-defined learning curve, and is easy to learn, requiring a minimum of 50 patients to achieve expert competency (intraclass correlation coefficient $>0.9$ ) in groups with varying levels of baseline skill over a period of 3 months [21]. Other studies have suggested an even shorter learning curve [22]. Prior background knowledge in echocardiography is an influential factor affecting the attainment of inter-observer reproducibility and time efficiency [21]. In contrast, short-axis strain analysis of global circumferential stain is more difficult and expert level was not achieved by the end of the study. Radial strain is inherently highly variable and probably should not be used outside of research settings.

In addition to the true error of the measurement, sources of variation may arise from patient characteristics (e.g., poor image quality due to body habitus and mastectomy is also a potential cause of underestimation), equipment (image acquisition and post-processing), and technical limitations on the part of the observer (particularly related to experience). The use of strain as a reliable physiologic marker is dependent on attention to detail. Imprecision in tracing the region of interest, inadequate tracking, failure to exclude the pericardium, and annulus are all potential source of variability and will lead to underestimation of GLS [23]. As GLS is load-dependent, some variation in sequential imaging may arise from differences in blood pressure - lower GLS measurements may be attributable to higher blood pressure measurements at the time of follow-up. The measurement of myocardial work accounts for loading conditions and may be a solution to this problem [24].

The process of establishing strain in an echocardiography laboratory should start with a concordance process between the personnel doing the measurements. Although different laboratories may show minor but important differences in strain measurements [25], once conformity has been defined 
between observers, drift in strain measurements seems to be small [22]. Nonetheless, evaluation of tracking and the shape of the strain curves is an essential aspect of using GLS (Fig. 1), which should not be simply gathered from the polar map display.

Application to Cardio-Oncology A number of observational studies concerning the use of myocardial deformation have been reported over the last decade. The reliability of these for detecting CTRCD was reviewed in a recent meta-analysis [26••]. Impaired GLS both at baseline and during treatment have a high-sensitivity and modest but variable specificity for identifying CTRCD (Table 2). Because of the inter-individual variation of GLS, using each patient as their own control is a preferable approach. Absolute changes of GLS between $2-3 \%$, and relative changes of between $10-15 \%$
Fig. 1 Underestimation of GLS due to technical difficulties. In this asymptomatic patient, biplane EF was $62 \%$ and GLS was reported to be $-14 \%$. Review of the polar map (a) shows heterogeneity of regional strain, disproportionately in the basal segments - this is an unusual pattern that should raise suspicion of artefact. Many of the strain curves (b) show an initial lengthening before shortening - this feature can be seen in the presence of scar, but again with be unusual in the setting of an apparently normal LV. Likewise, while heterogeneity of regional strain can be seen with diffuse processes, the magnitude of this variation is unusual. Revision of tracking demonstrated a normal GLS

Table 2 Sensitivity and specificity of GLS for predicting CTRCD. A, pretreatment GLS measurements; B, Absolute on-treatment GLS measurements; $\mathrm{C}$, Absolute difference in GLS; D, Relative change in GLS. Simplified from Oikonomou [26••]
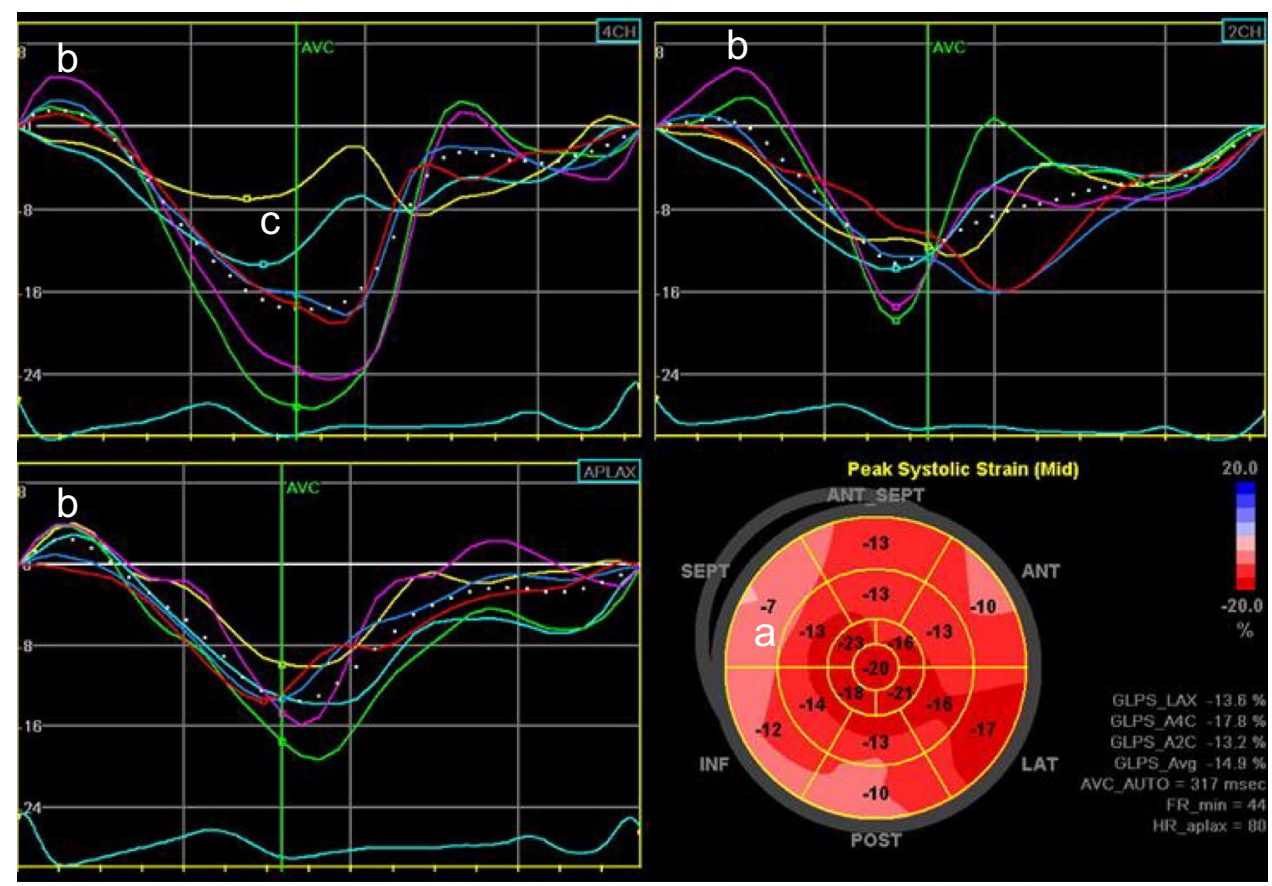

\begin{tabular}{llllll}
\hline & Author, date & Cancer type & Cutoff & Sensitivity & Specificity \\
\hline Pre-treatment GLS & Charbonnel, 2017 & Hematologic & $-20.0 \%$ & $83 \%$ & $72 \%$ \\
& Ali, 2016 & Hematologic & $-17.5 \%$ & $86 \%$ & $81 \%$ \\
Absolute on-treatment GLS & Milks, 2018 & Breast & $-19.0 \%$ & $64 \%$ & $83 \%$ \\
& Sawaya, 2012 & Breast & $-19.0 \%$ & $74 \%$ & $73 \%$ \\
& deAlmeida 2018 & Breast & $-16.6 \%$ & $80 \%$ & $95 \%$ \\
& Charbonnel, 2017 & Hematologic & $-17.5 \%$ & $83 \%$ & $72 \%$ \\
& Tang, 2016 & Breast & $-13.8 \%$ & $86 \%$ & $73 \%$ \\
& Paraskevaidis, 2017 & Hematologic & $-18.4 \%$ & $86 \%$ & $71 \%$ \\
& Guerra, 2016 & Breast & $-18.0 \%$ & $89 \%$ & $74 \%$ \\
& Portugal, 2017 & Breast & $-18.0 \%$ & $90 \%$ & $45 \%$ \\
& Negishi, 2013 & Breast & $-21.0 \%$ & $96 \%$ & $67 \%$ \\
& Fallah-Rad, 2011 & Breast & $2.00 \%$ & $80 \%$ & $81 \%$ \\
& Mornos, 2013 & Mixed & $2.77 \%$ & $80 \%$ & $73 \%$ \\
& Charbonnel, 2017 & Hematologic & $0.45 \%$ & $83 \%$ & $65 \%$ \\
Relative change in GLS (\%) diff in GLS & Milks, 2018 & Breast & $13.7 \%$ & $45 \%$ & $71 \%$ \\
& Negishi, 2013 & Breast & $11.0 \%$ & $67 \%$ & $95 \%$ \\
& Sawaya, 2012 & Breast & $10.0 \%$ & $78 \%$ & $79 \%$ \\
& Charbonnel, 2017 & Hematologic & $2.26 \%$ & $83 \%$ & $65 \%$ \\
& Baratta, 2013 & Mixed & $15.0 \%$ & $86 \%$ & $86 \%$ \\
& Florescu, 2014 & Breast & $9.0 \%$ & $86 \%$ & $81 \%$ \\
& Kang, 2013 & Hematologic & $15.9 \%$ & $86 \%$ & $75 \%$ \\
& Mornos, 2014 & Mixed & $13.7 \%$ & $88 \%$ & $71 \%$ \\
deAlmeida 2018 & Breast & $14.0 \%$ & $100 \%$ & $93 \%$ \\
\hline
\end{tabular}


have a sensitivity in the $80-90 \%$ range for the detection of CTRCD, with a specificity of $80 \%$, albeit with some variability (Table 2).

Because of the tighter confidence intervals of GLS compared to EF, earlier changes of myocardial function may be detected with GLS. Very often, this means that GLS is a window to subsequent change of EF, effectively providing an opportunity for earlier, and thereby hopefully more effective intervention.

Nonetheless, it needs to be acknowledged that it is hard to prove prognostic benefit of one cardioprotective strategy, compared to others. In the SUCCOUR trial, 331 patients were randomized between GLS guided and EF guided cardiac surveillance [27•]. Both groups had an approximately $3 \%$ reduction of $3 \mathrm{D}-\mathrm{EF}$, which is within the reliability of the test. However, the development of cardiotoxicity was more common (13.7\%) with EF guidance, compared with GLS guidance $(5.8 \%, p=0.02)$. This was primarily because 44 of 154 in the strain-guided group developed an abnormal response and were initiated on cardioprotective therapy, compared to 20 in the EF-guided group. The EF change in the 44 cardioprotection-treated, GLS guided patients was only $2.9 \%$, compared to $9.1 \%$ in the EF-guided group, consistent with later (and therefore less effective) initiation of therapy.

\section{Alternative Surveillance Strategies}

Assessment of Diastolic Function Diastolic dysfunction is a common manifestation of myocardial injury from various causes and can reasonably be expected to be a potential marker of CTRCD. Indeed, in a 2-year follow-up of 362 breast cancer patients, Upshaw et al. showed persistent worsening of diastolic function (manifest as reduced mitral annular velocities and reduced E/A ratio) [28]. Abnormal diastolic function was associated with a subsequent reduction of ejection fraction and worsening of GLS. This is a simple addition to the use of GLS and EF, but has the potential disadvantage that it is highly sensitive to other causes of myocardial injury, and therefore may be less specific than the other techniques.

Biomarkers Abnormal brain natriuretic peptide or highsensitivity troponin have been reported with CTRCD and would certainly be more feasible than imaging for surveillance. Unfortunately, the levels of these biomarkers are also quite variable, implying that sampling has to be done at exactly the right time in order to identify myocardial injury. Moreover, not only cardiotoxicity but also other circulatory stress may lead to release of these biomarkers. Thus, while proposed as a potential cornerstone of surveillance [29], their reliability remains debated.

Cardiac Magnetic Resonance (CMR) The most reliable assessment of LV volumes and ejection fraction is obtainable with CMR, and this has been shown to be effective in predicting outcomes [30]. The variability of CMR-LVEF is similar to echocardiographic 3D-EF or GLS, and less than echocardiographic 2D-EF [31]. CMR-based GLS is feasible, but probably not superior to echo-based GLS. The role of CMR tissue characterization remains to be defined. A concerning aspect of these parameters is their variability [32], and although markers of fibrosis, inflammation and edema should be able to identify the earliest stages of myocardial injury; they may not be sufficiently specific for use in guiding treatment.

\section{Response to Abnormal Test}

There are two potential responses to an abnormal test initiation of cardioprotection, or modifying or interrupting cardiotoxic therapy. The latter is very much a last option because it may have a detrimental effect on treatment of the cancer, and is rarely required unless the patient has developed overt heart failure or other steps have failed.

Four groups of medications have been used for cardioprotection. Dextrazoxane has been known to have a cardioprotective effect during anthracycline chemotherapy for nearly 50 years, but its uptake has always been impeded by concerns that it may limit the efficacy of anthracyclines, and thereby inhibit successful cancer remission. While its activity was traditionally attributed to protection from oxidative stress (through iron chelation), it seems more likely that its efficacy pertains to differential expression and/or regulation of topoisomerase II isoforms in cardiac and cancer cells, leading to the selective modulation of anthracycline action [33]. Because this agent is thought to provide protection from injury, the model would be uniform use from time of initiation of chemotherapy, rather than selective use based upon abnormal imaging findings (see below).

In a systematic review of 25 studies with neurohormonal blockade using angiotensin-converting enzyme inhibitors, angiotensin receptor blockers, and beta-blockers, Elghazawy [34] showed similar levels of efficacy between the groups, with a $2.4 \%$ absolute difference in EF between treated and untreated groups. Their effectiveness for preserving $\mathrm{EF}$ was similar immediately after therapy, and at 6 and 12 months following treatment. These medications are presumed to work by unloading the LV, as they do in heart failure, as well as potentially through antioxidant and other metabolic effects. 
The third group of agents that have been shown to be effective are statins, which are thought to limit the oxidative stress through which anthracyclines mediate cardiotoxicity. A recent meta-analysis of six studies [35] showed a $6 \%$ average difference in ejection fraction between treated and untreated groups, with a $60 \%$ odds reduction for developing cardiotoxicity. In a network meta-analysis, statins appeared to be more effective than neurohormonal blockade in protecting patients against a drop in EF [36].

The final class of cardio-protective treatments are modern heart failure drugs such as Entresto and SGLT2 inhibitors, but this evidence base is small and not yet ready for routine use.

The use of GLS surveillance is based on a fundamental decision to pursue selective rather than universal cardioprotection. A number of medications - angiotensin converting enzyme inhibitors, angiotensin receptor blockers, beta-adrenoceptor blockers and statins - have proven benefit in the prevention of cardiotoxicity. The use of cardioprotection in all patients at risk would be simpler than an imaging-based approach, and it avoids concerns about timing, test accuracy, and interpretation. However, the fundamental problem is that such an approach is dependent on acceptance that $80 \%$ of patients would receive this treatment unnecessarily, as cardiotoxicity develops in $<20 \%$. In the placebo groups of randomized controlled trials, the average change of EF - while variable - is most commonly $<5 \%$ [37]. Decision analytic models have shown that a selective approach is more cost-effective than universal approach [38].

If a selective (image-guided) strategy is used, there is some time urgency in the response to an abnormal imaging surveillance test. In a study of over 200 patients with anthracycline related CTRCD, Cardinale reported $64 \%$ to respond to ACE inhibitors and beta-blockade administered within 2 months, $<30 \%$ to respond to treatment administered between $2-4$ months, and $<10 \%$ to respond to treatment provided thereafter [39]. This responder status was important in predicting subsequent event-free survival, which was $<40 \%$ at 2 years in non-responders $(\mathrm{EF}<50 \%$ and $\triangle \mathrm{EF}<$ $10 \%)$ and partial responders ( $\mathrm{EF}<50 \%$ and $\Delta \mathrm{EF} \geq 10 \%)$.

\section{Conclusion}

Anthracycline chemotherapy continues to carry a risk of myocardial dysfunction and heart failure. While neurohormonal antagonists and statins reduce the risk of cancer treatment-related cardiac dysfunction (CTRCD), the frequency of this problem is too low to justify the uniform use of these cardioprotective therapies. Consequently, most centers use a risk-guided strategy of cardioprotection - most commonly using 2D-EF. The problem is that 2D-EF measurements have broad confidence intervals, so substantial changes are required to designate the presence of LV dysfunction. GLS provides a more reliable and reproducible means of assessing global cardiac function, and observational studies and a randomized trial have shown the most reliable approach is to use a $10-15 \%$ relative change of GLS with therapy. GLS is a feasible, robust, and reproducible technique that requires limited training and is ready for wide adoption.

Funding Open Access funding enabled and organized by CAUL and its Member Institutions. This study was supported in part by a Project Grant (1149692) from the National Health and Medical Research Council, Canberra, Australia.

\section{Declarations}

Conflict of Interest Thomas H. Marwick has received a research grant in support of the core laboratory of the SUCCOUR trial from General Electric Medical Systems.

Open Access This article is licensed under a Creative Commons Attribution 4.0 International License, which permits use, sharing, adaptation, distribution and reproduction in any medium or format, as long as you give appropriate credit to the original author(s) and the source, provide a link to the Creative Commons licence, and indicate if changes were made. The images or other third party material in this article are included in the article's Creative Commons licence, unless indicated otherwise in a credit line to the material. If material is not included in the article's Creative Commons licence and your intended use is not permitted by statutory regulation or exceeds the permitted use, you will need to obtain permission directly from the copyright holder. To view a copy of this licence, visit http://creativecommons.org/licenses/by/4.0/.

\section{References}

Papers of particular interest, published recently, have been highlighted as:

- Of importance

$\bullet$ Of major importance

1. Coleman MP, Quaresma M, Berrino F, et al. Cancer survival in five continents: a worldwide population-based study (CONCORD). Lancet Oncol. 2008;9:730-56.

2. Felker GM, Thompson RE, Hare JM, et al. Underlying causes and long-term survival in patients with initially unexplained cardiomyopathy. N Engl J Med. 2000;342:1077-84.

3. Chung R, Ghosh AK, Banerjee A. Cardiotoxicity: precision medicine with imprecise definitions. Open Heart. 2018;5:e000774.

4. Nolan MT, Marwick TH, Plana JC, et al. Effect of traditional heart failure risk factors on myocardial dysfunction in adult survivors of childhood cancer. JACC Cardiovasc Imaging. 2018;11:1202-3.

5. Hurtado-de-Mendoza D, Loaiza-Bonilla A, Bonilla-Reyes PA, Tinoco G, Alcorta R. Cardio-Oncology: Cancer Therapy-related Cardiovascular Complications in a Molecular Targeted Era: New Concepts and Perspectives. Cureus. 2017;9:e1258. 
6. Ewer MS, Ewer SM. Cardiotoxicity of anticancer treatments: what the cardiologist needs to know. Nat Rev Cardiol. 2010;7:564-75.

7. Von Hoff DD, Layard MW, Basa P, et al. Risk factors for doxorubicin-induced congestive heart failure. Ann Intern Med. 1979;91:710-7.

8. Mulrooney DA, Yeazel MW, Kawashima T, et al. Cardiac outcomes in a cohort of adult survivors of childhood and adolescent cancer: retrospective analysis of the Childhood Cancer Survivor Study cohort. BMJ. 2009;339:b4606.

9. Ezaz G, Long JB, Gross CP, Chen J. Risk prediction model for heart failure and cardiomyopathy after adjuvant trastuzumab therapy for breast cancer. J Am Heart Assoc. 2014;3:e000472.

10. Rushton M, Johnson C, Dent S. Trastuzumab-induced cardiotoxicity: testing a clinical risk score in a real-world cardio-oncology population. Curr Oncol. 2017;24:176-80.

11. Dobson R, Ghosh AK, Ky B, et al. BSE and BCOS guideline for transthoracic echocardiographic assessment of adult cancer patients receiving anthracyclines and/or trastuzumab. JACC CardioOncol. 2021;3:1-16.

12. Otterstad JE, Froeland G, St John SM, Holme I. Accuracy and reproducibility of biplane two-dimensional echocardiographic measurements of left ventricular dimensions and function. Eur Heart J. 1997;18:507-13.

13. Pellikka PA, She L, Holly TA, et al. Variability in ejection fraction measured by echocardiography, gated single-photon emission computed tomography, and cardiac magnetic resonance in patients with coronary artery disease and left ventricular dysfunction. JAMA Netw Open. 2018;1:e181456.

14. Echocardiographic Normal ranges meta-analysis of the left heart c. ethnic-specific normative reference values for echocardiographic LA and LV Size, LV mass, and systolic function: the EchoNoRMAL study. JACC Cardiovasc Imaging. 2015;8:656-65.

15. Thavendiranathan P, Liu S, Verhaert D, et al. Feasibility, accuracy, and reproducibility of real-time full-volume 3D transthoracic echocardiography to measure LV volumes and systolic function: a fully automated endocardial contouring algorithm in sinus rhythm and atrial fibrillation. JACC Cardiovasc Imaging. 2012;5:239-51.

16. Henry ML, Niu J, Zhang N, Giordano SH, Chavez-MacGregor M. Cardiotoxicity and cardiac monitoring among chemotherapy-treated breast cancer patients. JACC Cardiovasc Imaging. 2018;11:1084-93.

17. Chavez-MacGregor M, Niu J, Zhang N, et al. Cardiac monitoring during adjuvant trastuzumab-based chemotherapy among older patients with breast cancer. J Clin Oncol. 2015;33:2176-83.

18. Flachskampf FA, Blankstein R, Grayburn PA, et al. Global longitudinal shortening: a positive step towards reducing confusion surrounding global longitudinal strain. JACC Cardiovasc Imaging. 2019;12:1566-7.

19. Farsalinos KE, Daraban AM, Unlu S, Thomas JD, Badano LP, Voigt JU. Head-to-head comparison of global longitudinal strain measurements among nine different vendors: the EACVI/ ASE inter-vendor comparison study. J Am Soc Echocardiogr. 2015;28:1171-1181, e2 Variation of GLS measurements between vendors is often proposed as a rational for noto adopting GLS. These studies show that after the evolution of software and adoption of common guidelines, this variation is less than many other common measurements.

20. D'Elia N, Caselli S, Kosmala W, et al. Normal global longitudinal strain: an individual patient meta-analysis. JACC Cardiovasc Imaging. 2020;13:167-9.
21. Chan J, Shiino K, Obonyo NG, et al. Left ventricular global strain analysis by two-dimensional speckle-tracking echocardiography: the learning curve. J Am Soc Echocardiogr. 2017;30:1081-90.

22. Negishi T, Negishi K, Thavendiranathan P, et al. Effect of experience and training on the concordance and precision of strain measurements. JACC Cardiovasc Imaging. 2017;10:518-22.

23. Negishi K, Negishi T, Haluska BA, Hare JL, Plana JC, Marwick TH. Use of speckle strain to assess left ventricular responses to cardiotoxic chemotherapy and cardioprotection. Eur Heart $\mathbf{J}$ Cardiovasc Imaging. 2014;15:324-31.

24. Russell K, Eriksen M, Aaberge L, et al. A novel clinical method for quantification of regional left ventricular pressure-strain loop area: a non-invasive index of myocardial work. Eur Heart J. 2012;33:724-33.

25. Khouri MG, Ky B, Dunn G, et al. Echocardiography core laboratory reproducibility of cardiac safety assessments in cardiooncology. J Am Soc Echocardiogr. 2018;31:361-371 e3.

26.• Oikonomou EK, Kokkinidis DG, Kampaktsis PN, et al. Assessment of prognostic value of left ventricular global longitudinal strain for early prediction of chemotherapy-induced cardiotoxicity: a systematic review and meta-analysis. JAMA Cardiol. 2019;4:1007-18. This landmark meta-analysis reviews the observational studies of GLS as a predictor of CTRCD. it shows that while individual measurements have predictive value, the change of measures from baseline is the most predictive of CTRCD, with sensitivity and specificity in the range of $80-90 \%$.

27. Thavendiranathan P, Negishi T, Somerset E, et al. Strain-guided management of potentially cardiotoxic cancer therapy. J Am Coll Cardiol. 2021;77:392-401 This is the first RCT comparing GLS to EF for the management of patients undergoing potentially cardiotoxic chemotherapy. The overall change of EF in both arms was within the limits of reproducibility. However, the proportion of patients with CTRCD was less in the GLS-guided arm, reflecting the recognition and treatment of subclinical $L V$ dysfunction.

28. Upshaw JN, Finkelman B, Hubbard RA, et al. Comprehensive assessment of changes in left ventricular diastolic function with contemporary breast cancer therapy. JACC Cardiovasc Imaging. 2020;13:198-210.

29. Curigliano G, Cardinale D, Suter T, et al. Cardiovascular toxicity induced by chemotherapy, targeted agents and radiotherapy: ESMO clinical practice guidelines. Ann Oncol. 2012;23 Suppl 7:vii155-66.

30. Thavendiranathan P, Wintersperger BJ, Flamm SD, Marwick TH. Cardiac MRI in the assessment of cardiac injury and toxicity from cancer chemotherapy: a systematic review. Circ Cardiovasc Imaging. 2013;6:1080-91.

31. Lambert J, Lamacie M, Thampinathan B, et al. Variability in echocardiography and MRI for detection of cancer therapy cardiotoxicity. Heart. 2020;106:817-23.

32. Altaha MA, Nolan M, Marwick TH, et al. Can quantitative cmr tissue characterization adequately identify cardiotoxicity during chemotherapy?: impact of temporal and observer variability. JACC Cardiovasc Imaging. 2020;13:951-62.

33. Vavrova A, Jansova H, Mackova E, et al. Catalytic inhibitors of topoisomerase II differently modulate the toxicity of anthracyclines in cardiac and cancer cells. PLoS One. 2013;8:e76676.

34. Elghazawy H, Venkatesulu BP, Verma V, et al. The role of cardio-protective agents in cardio-preservation in breast cancer patients receiving Anthracyclines +/- Trastuzumab: a Meta-analysis of clinical studies. Crit Rev Oncol Hematol. 2020;153:103006 
35. Kim J, Nishimura Y, Kewcharoen J, Yess J. Statin use can attenuate the decline in left ventricular ejection fraction and the incidence of cardiomyopathy in cardiotoxic chemotherapy recipients: a systematic review and meta-analysis. J Clin Med. 2021;10:3731.

36. Alizadehasl A, Ghadimi N, Kaveh S, et al. Prevention of anthracycline-induced cardiotoxicity: a systematic review and network meta-analysis. Int J Clin Pharm. 2021;43:25-34.

37. Jeyaprakash P, Sangha S, Ellenberger K, Sivapathan S, Pathan F, Negishi K. Cardiotoxic Effect of modern anthracycline dosing on left ventricular ejection fraction: a systematic review and metaanalysis of placebo arms from randomized controlled trials. J Am Heart Assoc. 2021;10:e018802.
38. Nolan MT, Plana JC, Thavendiranathan P, Shaw L, Si L, Marwick TH. Cost-effectiveness of strain-targeted cardioprotection for prevention of chemotherapy-induced cardiotoxicity. Int J Cardiol. 2016;212:336-45.

39. Cardinale D, Colombo A, Lamantia G, et al. Anthracyclineinduced cardiomyopathy: clinical relevance and response to pharmacologic therapy. J Am Coll Cardiol. 2010;55:213-20.

Publisher's Note Springer Nature remains neutral with regard to jurisdictional claims in published maps and institutional affiliations. 